\title{
Retrospective clinical case series study in 2017 identifies Plasmodiumknowlesi as most frequent Plasmodium species in returning travellers from Thailand to Germany
}

Guenter Froeschl ${ }^{1,2}$, Hans Dieter Nothdurft' ${ }^{1}$, Frank von Sonnenburg, ${ }^{1}$, Gisela Bretzel ${ }^{1}$, Roman Polanetz ${ }^{1}$, Inge Kroidl ${ }^{1,2}$, Michael

Seilmaier ${ }^{3}$, Hans Martin Orth ${ }^{4}$, Sabine Jordan5, Peter Kremsner6, Sabine Vygen-Bonnet7, Michael Pritsch ${ }^{1,2}$, Michael Hoelscher ${ }^{1,2}$, Camilla Rothe $\mathbf{R}^{1,2}$

1. Division of Infectious Diseases and Tropical Medicine, Medical Center of the University of Munich (LMU), Munich, Germany

2. German Center for Infection Research (DZIF), Partner Site Munich, Munich, Germany

3. Klinikum München Schwabing, Munich, Germany

4. Klinik für Gastroenterologie, Hepatologie und Infektiologie, Universitätsklinikum Düsseldorf, Düsseldorf, Germany

5. University Medical Center Hamburg-Eppendorf, 1st Medical Department, Division of Tropical Medicine and Infectious Diseases, Hamburg, Germany

6. Institut für Tropenmedizin, Reisemedizin und Humanparasitologie des Universitätsklinikums Tübingen, Tübingen, Germany

7. Robert Koch-Institute, Department of Infectious Disease Epidemiology, Unit of Gastrointestinal Infections, Zoonoses and Tropical Infections, Berlin, Germany

Correspondence: Guenter Froeschl (froeschl@lrz.uni-muenchen.de)

Froeschl Guenter, Nothdurft Hans Dieter, von Sonnenburg Frank, Bretzel Gisela, Polanetz Roman, Kroidl Inge, Seilmaier Michael, Orth Hans Martin, Jordan

Sabine, Kremsner Peter, Vygen-Bonnet Sabine, Pritsch Michael, Hoelscher Michael, Rothe Camilla. Retrospective clinical case series study in 2017 identifies

Plasmodiumknowlesi as most frequent Plasmodium species in returning travellers from Thailand to Germany. Euro Surveill. 2018;23(29):pii=1700619. https://doi. org/10.2807/1560-7917.ES.2018.23.29.1700619

Febrile illnesses are common in travellers returning from south-east Asia. However, malaria is a rare diagnosis in this population. A series of Plasmodium knowlesi infections was noted in German travellers returning from Thailand since 2012. Infectious disease and tropical medicine facilities registered by the German Society for Tropical Medicine and International Health were contacted in March 2017, and asked to report previous $P$. knowlesi cases. In addition, surveillance data from the Robert Koch-Institute were analysed. The facilities reported a total of six $P$. knowlesi-positive cases, all were returning travellers from Thailand. The $P$. knowlesi-positive cases made up 6/9 of all diagnosed malaria cases imported from Thailand in the time period 2012 to 2017. In 4/5 of cases where a malaria rapid diagnostic test had been applied it revealed a negative result. $P$. knowlesi is an important differential diagnosis in travellers returning from south-east Asia with itineraries that include Thailand. This study highlights the importance of this Plasmodium species in this patient subgroup. Whenever malaria is suspected in a returning traveller from Thailand, $P$. knowlesi should be taken into consideration and a differential PCR be executed as currently the unequivocal diagnosis of $P$. knowlesi is based on nuclear amplification techniques.

\section{Background}

Germany is currently the second leading country worldwide in numbers of international travels with about 84 million international travel departures in 2015, most of them for leisure purposes [1]. About one in seven international travellers to overseas from Germany suffers from a medical condition during their travel, and among those affected, about one quarter require some kind of medical assistance [2]. Malaria remains a relevant differential diagnosis in febrile patients arriving from tropical and subtropical areas, with 1,068 imported malaria cases reported in Germany in 2015 [3]. Thailand is a favourite travel destination for German travellers with 761,000 arrivals from Germany in 2015. In the same year, Thailand reported almost 30 million international arrivals in total [4].

Malaria is considered hypoendemic in Thailand with a reported total of 14,755 confirmed cases and 33 deaths in a total population of about 68 million in 2015 (incidence: 21 cases/100,000). However, the estimated true incidence of malaria infections is expected to be much higher at an annual incidence rate of 176 cases per 100,000 population [5].

The German guidelines for malaria prevention in travellers are issued annually by the German Society for Tropical Medicine and International Health (DTG). DTG recommends carrying an anti-malarial standby 
Cases of Plasmodium knowlesi infections reported in Germany, 2012-2017

\begin{tabular}{|c|c|c|c|c|c|c|c|c|}
\hline $\begin{array}{l}\text { Case } \\
\text { ID }\end{array}$ & $\begin{array}{l}\text { Date of } \\
\text { admission }\end{array}$ & $\begin{array}{l}\text { Age in } \\
\text { years }\end{array}$ & Sex & $\begin{array}{l}\text { Level } \\
\text { of } \\
\text { care }\end{array}$ & RDT & $\begin{array}{c}\text { Smear } \\
\text { parasitaemia }^{a}\end{array}$ & $\begin{array}{c}\text { Nuclear } \\
\text { amplification } \\
\text { techniques }\end{array}$ & Treatment \\
\hline 1 & January 2012 & 54 & Male & IPD & Not done & $0.01 \%$ & $\begin{array}{l}\text { Sequencing } \\
\text { positive }^{\text {b }}\end{array}$ & Atovaquone/proguanil \\
\hline 2 & January 2013 & 55 & Female & IPD & $\begin{array}{l}\text { BinaxNOW, pan- } \\
\text { aldolase (T2 band) } \\
\text { positive }\end{array}$ & $0.2 \%$ & PCR positive ${ }^{c}$ & $\begin{array}{l}\text { Artesunate followed by } \\
\text { Artemether/lumefantrine }\end{array}$ \\
\hline 3 & $\begin{array}{c}\text { December } \\
2013\end{array}$ & 73 & Male & ICU & BinaxNOW negative & $3 \%$ & PCR positive ${ }^{c}$ & Quinine/doxycycline \\
\hline 4 & $\begin{array}{c}\text { December } \\
2014\end{array}$ & 52 & Female & IPD & BinaxNOW negative & $1 \%$ & PCR positive ${ }^{c}$ & Artemether/lumefantrine \\
\hline 5 & February 2015 & 42 & Male & IPD & BinaxNOW negative & $0.02 \%$ & PCR positive ${ }^{c}$ & Atovaquone/proguanil \\
\hline 6 & January 2017 & 45 & Male & OPD & BinaxNOW negative & $0.0002 \%$ & PCR positive ${ }^{c}$ & Atovaquone/proguanil \\
\hline
\end{tabular}

ICU: intensive care unit; IPD: in-patient department; OPD: out-patient department; RDT: rapid diagnostic test.

a Percentages of infected erythrocytes are presented.

b Sequencing of small subunit ribosomal RNA, Basic Local Alignment Search Tool analysis of the obtained sequence, yields a $96 \%$ match with the respective $P$. knowlesi sequence.

${ }^{c}$ A PCR with primers specific for $P$. knowlesi was conducted.

medication when travelling to Thai regions bordering Cambodia, Laos and Myanmar only, and only if urban settings with a presumably adequate medical infrastructure are left behind [6].

The Plasmodium species mentioned in the latest 2016 World Malaria Report as being prevalent in Thailand are $P$. falciparum ( $41.8 \%$ of all specified cases) and $P$. vivax (58.2\%) [5]. Previous publications in 2011 and 2015 have indicated occasional cases of $P$. knowlesi infections in the resident population of Thailand $[7,8]$. In addition, on rare occasions, $P$. knowlesi infections in German travellers returning from Thailand have been reported [9-13]. Published studies, mostly on the basis of individual case reports, from other European countries, such as Finland, France, Spain, Sweden and the Netherlands, have also reported on importations of $P$. knowlesi infections from south-east Asia in returning travellers, indicating the diagnosis of a $P$. knowlesi infection as a generally rare event. The advent of occasional $P$. knowlesi identifications in returning European travellers seems to have started about a decade ago [14-20]. Of note, a recent analysis by the GeoSentinel Network, a global network for surveillance of travel-related illnesses, which included data from 29 countries, only reported three cases between 2003 and 2016, all imported from south-east Asia, whereby the explicit countries of acquisition were not indicated [21].

Malaria caused by $P$. knowlesi is a zoonotic infection endemic to south-east Asia, with a primary reservoir in macaques. The geographical distribution of $P$. knowlesi is therefore linked to the presence of its primary hosts. Humans can be infected occasionally, especially in areas where human settlements are advancing into habitats of the reservoir host, namely forested areas. Investigations on archived blood samples in Malaysia have revealed that the large majority of cases previously classified by microscopy as $P$. malariae infections were in fact $P$. knowlesi infections [22]. The parasite species has a short replication cycle of only 24 hours and can thus rapidly generate high parasite loads [23]. More frequently than in infections with $P$. malariae, $P$. vivax and $P$. ovale, $P$. knowlesi causes severe courses of disease that resemble $P$. falciparum malaria. In particular, respiratory and renal complications have been reported [16].

Upon noticing a series of $P$. knowlesi cases in people returning from Thailand to Germany, this study was conceived in order to investigate the relevance of this parasite as a causative agent for febrile conditions in such travellers.

\section{Methods}

\section{Questionnaire}

A digital questionnaire was compiled in the format of a case report file (Microsoft Word). For each reported $P$. knowlesicase, one questionnaire was to be completed by the facility (as described below) that identified the case. Questions included age, sex, purpose of travel, travel itinerary, date of return, date of presentation, clinical presentation, means of diagnosis employed, highest level of care, treatment regimen and clinical course. In addition, details were requested on the total number of malaria cases in the respective facility for the respective reporting year in patients returning from the same country from which the reported $P$. knowlesi case had returned. 
Map of Thailand with travel destinations of cases of Plasmodium knowlesi infections, 2012-2017

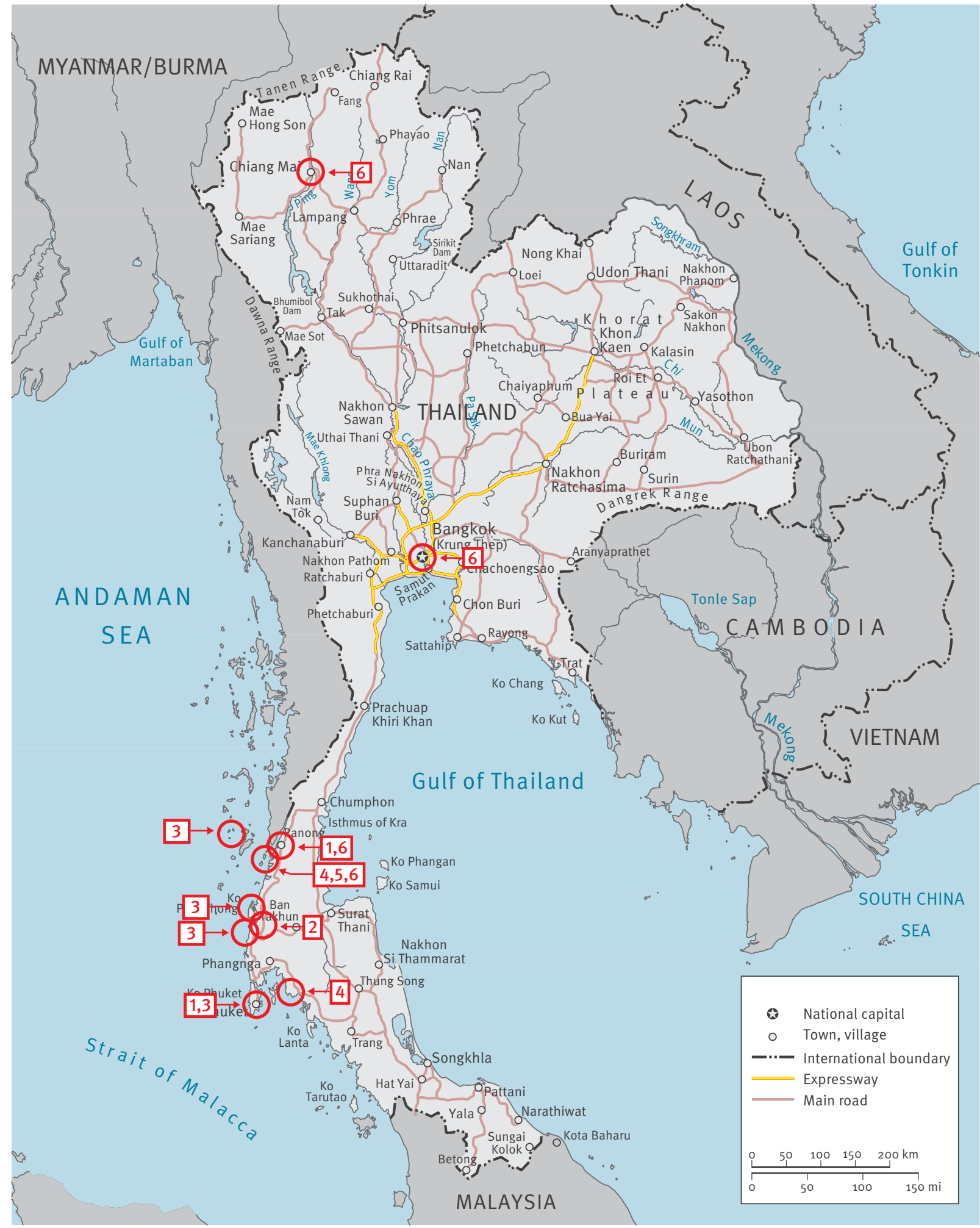

This map is adapted from a map by the Department of Field Support, Cartographic Section of the United Nations [32]. Encircled in red are locations of stay as indicated by the reported cases. Numbers in arrowed boxes are indicating case IDs as outlined in the Table. 


\section{Data sources}

The DTG is a reference institution in the field of travel medicine in Germany with annual publications on malaria risk profiles for worldwide travel destinations and guidelines on prevention and treatment of malaria. The society lists all major infectious diseases and tropical medicine facilities in Germany. The complete list of 17 facilities was contacted on 16 March 2017 with an invitation to participate in the study by reporting whether $P$. knowlesi cases had been diagnosed previously at each respective facility, and if so, to complete one questionnaire per $P$. knowlesi case. Furthermore, corresponding authors of already published cases (who were found as further described) were directly contacted. The last completed form was returned on 23 May 2017.

In addition, a literature search of articles in English language in PubMed using the keywords 'knowlesi' AND 'Germany' was conducted. Malaria in Germany is mandatorily reported to the national public health institute (the Robert Koch-Institute; RKI). The archive and surveillance data of the RKI were searched to potentially identify additional cases [24].

\section{Results}

\section{Case finding}

In total 17 facilities were contacted, including the facility of the study coordinators. Sixteen of the 17 facilities replied and provided completed questionnaires if cases had been observed. Eleven facilities indicated never having diagnosed any case of $P$. knowlesi infections. Four facilities reported one case each and one facility reported two respective cases, resulting in a total of six $P$. knowlesi cases. The earliest case ever reported in Germany was in 2012 and all six cases had travelled to Thailand. A literature search revealed only cases who were also reported directly by the participating facilities [9-13].

An investigation of the archive and the surveillance data of the RKI provided a total of nine notified malaria cases across all Plasmodium species that were imported from Thailand to Germany in the time period 2012 to 2017 as denominator. As part of these nine, all six $P$. knowlesi cases who were reported by the specialised facilities through this study could also be retrieved in the RKI surveillance data. In addition, one $P$. knowlesi case with missing information on travel purpose, destination and probable country of infection was revealed by the surveillance data; this case could not be included in the analysis.

\section{Epidemiological and demographic description of cases}

The reported six cases comprised four males and two females, the age range was 42 to 73 years and all cases were German citizens. The earliest case was from 2012 and had travelled to Thailand. Six further cases of $P$. knowlesiinfections subsequently occurred, five of which had also travelled to Thailand and one with travel information missing, as mentioned above. Cases are hereafter referred to by case identification numbers, as outlined in the Table.

Tourism was the main purpose of travel in five of six cases, one case reported to have visited friends and relatives (case 1). All travels took place in the months of November to February.

From a more detailed investigation of the respective travel itineraries, it was noted that all cases had travelled to the southern Thai provinces of Ranong, PhangNga and Surat Thani (Figure).

It has to be pointed out that the island of (Little) Koh Chang in the Andaman Sea, which should not be confused with the larger island of Koh Chang in the Gulf of Thailand, was mentioned as a destination by three of the travellers (cases 4,5,6), and for all three this island was the location with a considerably longer duration of stay. Only one patient (case 3) also travelled outside Thailand during the itinerary; however, his destination in neighbouring Myanmar was the island of Macleod, which lies in the immediate vicinity of Ranong Province. In addition to the above named provinces, patients mentioned Bangkok as city of entry including just a brief stop-over, and one patient (case 6) also travelled to the northern city of Chiang Mai before passing the largest part of the stay in the southern province of Ranong (Figure).

\section{Case clinical presentation, diagnostics and management}

All cases presented with fever (temperature $\geq 38.0^{\circ} \mathrm{C}$ ) as the leading symptom. The lag-time between return from travel and presentation at the facilities was between 1 and 23 days. In all cases a positive blood smear led to the diagnosis of a Plasmodium infection. In 5/6 cases, the microscopy result was initially reported as presence of $P$. falciparum or $P$. malariae, but also morphological resemblance to $P$. vivax and $P$. ovale was reported in two respective cases. Co-infections by multiple species were reported in the initial smear results in three of the six cases. The parasite load was in the range between $0.0002 \%$ and $3 \%$. The parasite load was particularly low in case 6 , where the microscopy result that led to further investigations was based on a single gametocyte in a total of 100 investigated fields at $400 x$ magnification. In five of the six cases (cases 2-6) a rapid diagnostic test (RDT) for malaria was conducted. All facilities used the BinaxNOW Malaria assay (Alere, Scarborough, Maine, USA). In four of five RDTs conducted the test revealed a negative result. Only one facility reported a positive $\mathrm{T} 2$ band (case 2), corresponding to the pan-plasmodial aldolase (Table). According to the BinaxNOW Malaria product specifications a positive $T_{2}$ band with a negative $T_{1}$ band is interpreted as a mono- or mixed infection by $P$. vivax, $P$. ovale or $P$. malariae, in the absence of $P$. falciparum. All six reported cases were confirmed 
to be $P$. knowlesi by nuclear amplification techniques. For case 1 the small subunit ribosomal RNA sequence was amplified and the sequence was further analysed by Basic Local Alignment Search Tool (BLAST). This yielded $96 \%$ identitity with sequencing data from $P$. knowlesi [13]. For the other cases a differential PCR with primers for $P$. knowlesi was conducted. Five of the six cases, were hospitalised. In two cases the World Health Organization (WHO) criteria for severe malaria were fulfilled [25]. In one case admission to an intensive care unit was necessary due to severe presentation with renal and respiratory insufficiency, acidosis and reduced vigilance (case 3 ). This case was at the same time the one with the highest parasitaemia of $3 \%$ in this case series. A second case of complicated $P$. knowlesi malaria was treated with renal impairment; however, in this case intensive care was not pursued (case 2). Treatment was in all cases initiated prior to the conclusive diagnosis of $P$. knowlesi infection, and corresponded with the results of initial microscopy. As the applied regimens were assumed to be effective also in $P$. knowlesi infections, no change in treatment regimen had to be executed in any of the cases (Table).

The treatment outcome was considered successful in all cases. The time until improvement of symptoms was indicated as 1, 2 and 3 days after commencement of anti-malarial treatment, for three of the reporting facilities where this information was retrievable from files. No recrudescence was reported in any of the cases.

\section{Proportion of Plasmodium knowlesi cases among malaria cases imported from Thailand to Germany}

The five facilities reporting $P$. knowlesi cases were asked to indicate the total number of malaria cases for the same travel destination from which the index case had imported the $P$. knowlesi infection (in all cases Thailand) for the respective year of diagnosis of the $P$. knowlesi case. In total, seven cases of malaria were reported by the facilities in returning travellers from Thailand in the respective years, including the six cases of $P$. knowlesi infections. Only one facility indicated one additional case other than $P$. knowlesi in a returning traveller from Thailand for the year 2014 . The proportion of $P$. knowlesi infections among all malaria cases from Thailand in the reporting facilities for the reported years is therefore amounting to 6 of 7. When the total number of nine malaria cases who were reported to the RKI as imported from Thailand to Germany in the time period 2012 to 2017 is taken as denominator, the proportion of $P$. knowlesi infections amounts to 6 of 9 of all imported cases from Thailand. The remaining cases were two $P$. vivax and one $P$. falciparum infection.

\section{Discussion and conclusions}

Malaria remains a very low risk for German travellers to the favourite travel destination Thailand. In 2015, a total of 761,000 travellers from Germany departed for this country. The RKI reported a total number of
1,068 imported malaria cases to Germany for 2015 . Only two of these were imported from Thailand [3]. In the entire time period from 2012 to 2017, only a total of nine malaria cases were reported to the national public health institute as imported from Thailand. The facilities participating in this study, which are among the largest facilities in Germany specialised in the field of infectious diseases and tropical medicine, identified six infections that were due to $P$. knowlesi. This species is not reported as prevalent in Thailand in the latest World Malaria Report of 2016 [5]. Given the finding, that in most cases a Plasmodium species other than $P$. knowlesi was initially morphologically indicated, and that the common range of anti-malarials applied are also active against $P$. knowlesi, we assume that imported $P$. knowlesi infections may frequently remain undetected. It is of note that resemblance in $P$. knowlesi morphology may be indicative of the stage in replication cycle, as early stages have been reported to rather resemble $P$. falciparum, whereas later stages more frequently show features of $P$. malariae [26].

In addition, more and more non-specialist institutions rely for their primary diagnosis of malaria on RDTs, which are highly sensitive for $P$. falciparum malaria but frequently fail to detect non-falciparum species [27]. In this study, the specialised facilities executed an RDT in five of the six $P$. knowlesi cases. In all cases the RDT used was the BinaxNOW Malaria. Only one test revealed a positive result for the aldolase antigen only. The BinaxNOW has been designed and validated by the manufacturer for $P$. falciparum and $P$. vivax infections only. The $\mathrm{T}_{1}$ band represents the histidine-rich-protein II, which is a specific antigen for $P$. falciparum, whereas the $\mathrm{T}_{2}$ band represents the panplasmodial aldolase antigen. For $P$. falciparum the manufacturer indicates an overall sensitivity of $95.3 \%$ and a specificity of $94.2 \%$; the test performance is lower in $P$. vivax infections, with an overall sensitivity of $68.9 \%$ (specificity $99.8 \%$ ). The product information by the manufacturer indicates that the RDT is also able to detect $P$. ovale and $P$. malariae but for these species the clinical performance has not been adequately established. P. knowlesi is not mentioned in the test specifications [28]. A study conducted in Malaysia reported low sensitivity of RDTs that are based on the panplasmodial aldolase for all Plasmodium species, with the lowest sensitivity in $P$. knowlesi infections at $23 \%$. Although sensitivity generally increased with parasite load, only $45 \%$ of highly parasitaemic patients with a $P$. knowlesi infection ( $>10,000$ parasites $/ \mu \mathrm{L}$ ) revealed a positive result [27]. This finding is corroborated by the results of this study where one positive pan-plasmodial aldolase test was found in five patients. RDTs with a pan-plasmodial lactate dehydrogenase component generally seem to perform with a higher sensitivity for all species including $P$. knowlesi, but even there one in four $P$. knowlesi infections remains undetected by the assay [27]. Of note, three of the six cases reported in this study had a low parasitaemia of $10.1 \%$ infected 
erythrocytes, which contributes to impaired test performance even more.

In settings where malaria detection is depending on RDT performance, a possible case of $P$. knowlesi malaria is likely to remain undetected or to be complicated by late diagnosis. Severe courses of infection in immunologically naive individuals are assumed to be less frequent in $P$. knowlesi than in $P$. falciparum infections, but more frequent than in $P$. malariae, $P$. vivax or $P$. ovale, which corroborates the importance of a valid species differentiation. In this study, two of six cases fulfilled criteria for severe malaria.

Since malaria still is a possible aetiology for febrile conditions in returning travellers with a potentially severe clinical course, the findings of this study suggest that $P$. knowlesi may need to be added to the risk profile of Thailand. The high proportion of $P$. knowlesi infections in the investigated population of this study (6/9) demands that once the diagnosis of a malaria infection is established in a traveller returning from Thailand (or indeed elsewhere from south-east Asia), a differential malaria PCR that comprises $P$. knowlesi should be executed, irrespective of the species differentiation through microscopy, and irrespective of an RDT result. In addition, in cases with a persistent suspicion of a malaria infection but with negative smear and RDT results, a differential PCR comprising $P$. knowlesi should be considered as a means of diagnosis of a possible $P$. knowlesi infection presenting with a low parasitaemia. However, the extremely low incidence of only two cases of malaria across all species in an annual 761,000 travels from Germany to Thailand may provoke a reconsideration of current travel recommendations with regard to malaria prevention, especially in light of reports of inadequate use of anti-malarial standby medication [29].

The findings of this study are expected to be of equal relevance to other European countries with comparable tourism profiles of their citizens. As we previously highlighted, identification of $P$. knowlesi remains currently a rare event for countries such as Finland, Sweden, Spain and France. However, as the overall incidence of Plasmodium infections across all species in European travellers returning from south-east Asia is largely declining [30] the consideration of $P$. knowlesi infections becomes important based on our and earlier findings.

There are some limitations to this study. A selection bias could have occurred, as only infectious disease and tropical medicine facilities registered by the DTG were contacted. A relevant number of cases of malaria are detected outside these facilities in Germany. In addition, the authors suspect a number of unrecognised - or more specifically misclassified - cases of $P$. knowlesi infections, as in many cases of febrile illnesses in returning travellers from Thailand the necessary differential PCR will not have been executed.
The authors are convinced that a number of the cases, who were previously classified as infections by especially $P$. malariae and $P$. falciparum from southeast Asia, were actually infected by $P$. knowlesi.

The importance of considering $P$. knowlesi in malaria control efforts in south-east Asia has been pointed out in a recent publication by Barber et al. [31]. Taking travellers as sentinels, our cases series may highlight an, as yet, under-addressed public health problem in some parts of Thailand. Further PCR-based studies in targeted areas of Thailand should be of public health interest to find out which share $P$. knowlesi infection takes in human as well as in simian malaria.

\section{Acknowledgements}

The authors would like to thank their colleagues from all German institutions in infectious diseases and tropical medicine as listed by the DTG for having completed the report forms without having had P. knowlesi cases so far. Their contribution has been equally important to this study just as those from institutions that were able to report cases.

Funding: The work presented in this manuscript has exclusively been funded by institutional funds of the authors.

\section{Conflict of interest}

None declared.

Authors' contributions

GF and CR conceived the study, were responsible for data collection and wrote the manuscript; GF, HDN, FvS, GB, MP, $\mathrm{MH}$ and CR conducted the data analysis; RP, IK, MS, HMO, SJ, PK and SVB collected data and filled case report forms. All authors critically revised and approved the final version of the manuscript.

\section{References}

1. World Bank (WB). International tourism, number of departures. Washington, DC: WB; 2017. [Accessed 19 Aug 2017]. Available from: http://data.worldbank.org/indicator/ST.INT. DPRT?locations $=\mathrm{DE}$

2. Fleck $S$, Jäger $H$, Zeeb $H$. Travel and health status: a survey follow-up study. Eur J Public Health. 2006;16(1):96-100. https://doi.org/10.1093/eurpub/cki144 PMID: 16030132

3. Robert Koch-Institute (RKI). Infektionsepidemiologisches Jahrbuch für 2015. [Infection Epidemiological Yearbook for 2015]. Berlin: RKI; 2016. German. Available from: https:// www.rki.de/DE/Content/Infekt/Jahrbuch/Jahrbuch_2015. pdf?__blob=publicationFile

4. Thailand Department of Tourism. Nonthaburi; 2015. [Accessed 19 Aug 2017]. Available from: http://tourism2.tourism.go.th/ home/details/11/221/24710

5. World Health Organization (WHO). World Malaria Report 2016. Geneva: WHO; 2016.

6. Deutsche Gesellschaft für Tropenmedizin und Internationale Gesundheit (DTG) e. V. (DTG). Hamburg: DTG; 2017. [Accessed 19 Aug 2017]. Available from: http://dtg.org/empfehlungenund-leitlinien/empfehlungen/malaria.html

7. Jongwutiwes S, Buppan P, Kosuvin R, Seethamchai S, Pattanawong U, Sirichaisinthop J, et al. Plasmodium knowlesi Malaria in humans and macaques, Thailand. Emerg Infect Dis. 2011;17(10):1799-806. https://doi.org/10.3201/eid1710.110349 PMID: 22000348 
8. Nakaviroj S, Kobasa T, Teeranaipong P, Putaporntip $C$, Jongwutiwes $S$. An autochthonous case of severe Plasmodium knowlesi malaria in Thailand. Am J Trop Med Hyg. 2015;92(3):569-72. https://doi.org/10.4269/ajtmh.14-0610 PMID: 25535314

9. Kroidl I, Seilmaier M, Berens-Riha N, Bretzel G, Wendtner C Löscher T. "Affenmalaria” nach Thailandreise. [Monkey malaria (Plasmodium knowlesi infection) after travelling to Thailand]. Dtsch Med Wochenschr. 2015;140(11):815-7. PMID: 26080720

10. Orth H, Jensen BO, Holtfreter MC, Kocheril SJ, Mallach S, MacKenzie C, et al. Plasmodium knowlesi infection imported to Germany, January 2013. Euro Surveill. 2013;18(40):20603. https://doi.org/10.2807/1560-7917.ES2013.18.40.20603 PMID: 24128698

11. Mackroth MS, Tappe D, Tannich E, Addo M, Rothe C. RapidAntigen Test Negative Malaria in a Traveler Returning From Thailand, Molecularly Diagnosed as Plasmodium knowlesi. Open Forum Infect Dis. 2016;3(1):ofw039. https://doi. org/10.1093/ofid/ofw039 PMID: 27006963

12. Seilmaier M, Hartmann W, Beissner M, Fenzl T, Haller C, Guggemos W, et al. Severe Plasmodium knowlesi infection with multi-organ failure imported to Germany from Thailand/Myanmar. Malar J. 2014;13(1):422. https://doi. org/10.1186/1475-2875-13-422 PMID: 25367021

13. Ehrhardt J, Trein A, Kremsner P, Frank M. Plasmodium knowlesi and HIV co-infection in a German traveller to Thailand. Malar J. 2013;12(1):283. https://doi.org/10.1186/1475-2875-12-283 PMID: 23941258

14. Ta TT, Salas A, Ali-Tammam M, Martínez MC, Lanza M, Arroyo $E$, et al. First case of detection of Plasmodium knowlesi in Spain by Real Time PCR in a traveller from Southeast Asia. Malar J. 2010;9(1):219. https://doi.org/10.1186/1475-2875-9219 PMID: 20663184

15. Kantele A, Marti H, Felger I, Müller D, Jokiranta TS. Monkey malaria in a European traveler returning from Malaysia. Emerg Infect Dis. 2008;14(9):1434-6. https://doi.org/10.3201/ eid1409.080170 PMID: 18760013

16. Kantele A, Jokiranta TS. Review of cases with the emerging fifth human malaria parasite, Plasmodium knowlesi. Clin Infect Dis. 2011;52(11):1356-62. https://doi.org/10.1093/cid/cir180 PMID: 21596677

17. Bronner U, Divis PC, Färnert A, Singh B. Swedish traveller with Plasmodium knowlesi malaria after visiting Malaysian Borneo. Malar J. 2009;8(1):15. https://doi.org/10.1186/1475-2875-8-15 PMID: 19146706

18. Berry A, Iriart X, Wilhelm N, Valentin A, Cassaing S, Witkowski $B$, et al. Imported Plasmodium knowlesi malaria in a French tourist returning from Thailand. Am J Trop Med Hyg. 2011;84(4):535-8. https://doi.org/10.4269/ajtmh.2011.10-0622 PMID: 21460005

19. van Hellemond JJ, Rutten M, Koelewijn R, Zeeman AM, Verweij JJ, Wismans PJ, et al. Human Plasmodium knowlesi infection detected by rapid diagnostic tests for malaria. Emerg Infect Dis. 2009;15(9):1478-80. https://doi.org/10.3201/ eid1509.090358 PMID: 19788819

20. Link L, Bart A, Verhaar N, van Gool T, Pronk M, Scharnhorst V. Molecular detection of Plasmodium knowlesi in a Dutch traveler by real-time PCR. J Clin Microbiol. 2012;50(7):2523-4. https://doi.org/10.1128/JCM.06859-11 PMID: 22573596

21. Angelo KM, Libman M, Caumes E, Hamer DH, Kain KC, Leder K, et al. GeoSentinel Network. Malaria after international travel: a GeoSentinel analysis, 2003-2016. Malar J. 2017;16(1):293. https://doi.org/10.1186/s12936-017-1936-3 PMID: 28728595

22. Lee KS, Cox-Singh J, Brooke G, Matusop A, Singh B. Plasmodium knowlesi from archival blood films: further evidence that human infections are widely distributed and not newly emergent in Malaysian Borneo. Int J Parasitol. 2009;39(10):1125-8. https://doi.org/10.1016/j. ijpara.2009.03.003 PMID: 19358848

23. Antinori S, Galimberti L, Milazzo L, Corbellino M. Biology of human malaria plasmodia including Plasmodium knowlesi. Mediterr J Hematol Infect Dis. 2012;4(1):e2012013. https://doi. org/10.4084/mjhid.2012.013 PMID: 22550559

24. Robert Koch-Institute (RKI). Berlin: RKI; 2017. [Accessed 19 Aug 2017]. Available from: http://www.rki.de/SiteGlobals/Forms/ Suche/serviceSucheForm.html? $\mathrm{nn}=2375194$ \& resourceld $=2390$ 936\&input_=2375194\&pageLocale $=$ de $\&$ searchEngineQueryStri $\mathrm{ng}=$ knowlesi\&submit. $x=0 \&$ submit. $y=0$

25. World Health Organization (WHO). Guidelines for the Treatment of Malaria. Geneva: WHO; 2015.

26. Lee KS, Cox-Singh J, Singh B. Morphological features and differential counts of Plasmodium knowlesi parasites in naturally acquired human infections. Malar J. 2009;8(1):73. https://doi.org/10.1186/1475-2875-8-73 PMID: 19383118
27. Barber BE, William T, Grigg MJ, Piera K, Yeo TW, Anstey NM. Evaluation of the sensitivity of a pLDH-based and an aldolasebased rapid diagnostic test for diagnosis of uncomplicated and severe malaria caused by PCR-confirmed Plasmodium knowlesi, Plasmodium falciparum, and Plasmodium vivax. J Clin Microbiol. 2013;51(4):1118-23. https://doi.org/10.1128/ JCM.03285-12 PMID: 23345297

28. Abbott. BinaxNOW Malaria. City: Abbott; 2017. Available from: https://www.alere.com/en/home/product-details/binaxnowmalaria.html

29. Vinnemeier CD, Rothe C, Kreuels B, Addo MM, Vygen-Bonnet $S$, Cramer JP, et al. Response to fever and utilization of standby emergency treatment (SBET) for malaria in travellers to Southeast Asia: a questionnaire-based cohort study. Malar J. 2017;16(1):44. https://doi.org/10.1186/s12936-017-1678-2 PMID: 28122576

30. Behrens RH, Carroll B, Hellgren U, Visser LG, Siikamäki $H$, Vestergaard LS, et al. The incidence of malaria in travellers to South-East Asia: is local malaria transmission a useful risk indicator? Malar J. 2010;9(1):266. https://doi. org/10.1186/1475-2875-9-266 PMID: 20920352

31. Barber BE, Rajahram GS, Grigg MJ, William T, Anstey NM. World Malaria Report: time to acknowledge Plasmodium knowlesi malaria. Malar J. 2017;16(1):135. https://doi.org/10.1186/ S12936-017-1787-y PMID: 28359340

32. Department of Field Support (DFS). Cartographic Section of the United Nations. Map of Thailand 2009.

\section{License and copyright}

This is an open-access article distributed under the terms of the Creative Commons Attribution (CC BY 4.0) Licence. You may share and adapt the material, but must give appropriate credit to the source, provide a link to the licence, and indicate if changes were made.

This article is copyright of the authors, 2018. 